\title{
Extensión universitaria y educación alimentaria nutricional en el nivel inicial hilos que tejen y construyen redes
}

\author{
University Extension and Nutritional Food Education at \\ the Initial Level: Threads That Weave and Build Networks \\ Extensão universitária e educação alimentar nutricional \\ no nível inicial: fios que tecem e formam redes
}

Luciana Avarese
Universidad Nacional del Litoral

Argentina

luciavar17@gmail.com

iD https://orcid.org/0000-0001-8620-8328

Carla Capovilla

Universidad Nacional del Litoral

Argentina

capovillacc@gmail.com

iD https://orcid.org/0000-0003-2789-7076

Nadina Boimvaser

Universidad Nacional del Litoral

Argentina

nadinboimvaser@gmail.com

iD https://orcid.org/0000-0002-4676-2778

Sandra Ravelli

Universidad Nacional del Litoral

Argentina

sravelli@fbcb.unl.edu.ar

http://orcid.org/0000-0001-7201-5963

Recibido: 31/07/2020 • Aceptado: 02/11/2020

Resumen. El siguiente artículo otorga voz a los diferentes actores involucrados en el Proyecto de Extensión de Interés Social (PEIS) En tus aventuras ¡frutas y verduras!: promoción del consumo en el nivel inicial, en el marco de un trabajo final de la Licenciatura en Nutrición de la Universidad Nacional del Litoral. 
Revista Universidad en Diálogo • Vol. 11, N. ${ }^{\circ}$ 1, Enero-Junio, 2021 • 157-178

ISSN 2215-2849 • EISSN: 2215-4752

URL: http://www.revistas.una.ac.cr/index.php/dialogo/index CORREO ELECTRÓNICO: universidadendialogo@una.cr DOI: http://doi.org/10.15359/udre.11-1.6

La publicación permite efectuar un análisis del impacto de la extensión a lo largo de tres años consecutivos, siendo la educación alimentaria nutricional el hilo conductor de cada actor involucrado.

A través de distintos recursos metodológicos se relevaron voces de niños y niñas del nivel inicial, de sus familias, de docentes, de personal directivo de las instituciones y, por último, de personas voluntarias extensionistas e investigadoras.

El PEIS impactó en las personas participantes, transformando a cada una de ellas, fortaleciendo saberes, estableciendo vínculos, aprendizajes, valores, recursos y estrategias, además de la reflexión sobre la actividad de extensión propiamente dicha.

Palabras clave: educación alimentaria nutricional, nivel inicial, valoración de impacto, voces participantes.

Abstract. This article gives voice to different actors involved in the Social Interest Extension Project (PEIS) titled "In your adventures, fruits and vegetables! Promotion of consumption at the initial level," within the framework of a final project of the bachelor's degree in Nutrition from the Universidad Nacional del Litoral.

The publication analyzes the impact of the university extension over three consecutive years, being nutritional food education the common thread for each actor involved. Through different methodological resources, children's voices from the initial level were surveyed, as well as those of their families, teachers, institutions' principals, and volunteer extensionists and researchers.

The PEIS impacted the different participants, transforming them, strengthening knowledge, establishing links, learnings, values, resources, and strategies, and reflecting on the extension activity itself.

Keywords: nutritional food education, initial level, impact assessment, participants'voices.

Resumo. O presente artigo destaca o papel dos diferentes participantes envolvidos no Projeto de Extensão de Interesse Social (PEIS) "Em suas aventuras, frutas e legumes! Promoção do consumo no nível inicial", no âmbito do projeto final do curso de Nutrição da Universidade Nacional do Litoral.

A publicação permite fazer uma análise do impacto da extensão em três anos consecutivos, tendo a educação alimentar e nutricional como fio condutor para cada ator envolvido.

Através de diferentes recursos metodológicos, foram coletadas as vozes das crianças do Jardim da infância, das suas famílias, dos professores, funcionários da administração escolar e, finalmente, voluntários e pesquisadores de extensão.

O PEIS teve um impacto significativo sobre os participantes, transformando cada um deles, fortalecendo o conhecimento, estabelecendo vínculos, aprendizados, valores, recursos e estratégias, além de refletir sobre a própria atividade de extensão.

Palavras-Chave: educação alimentar nutricional, nível inicial, avaliação de impacto, vozes dos participantes. 


\section{Introducción}

El siguiente artículo teje las voces de diferentes actores de la extensión universitaria que participaron y/o continúan participando en el Proyecto de Extensión de Interés Social (PEIS) "En tus aventuras ;frutas y verduras!: promoción del consumo en el nivel inicial", en el marco de un trabajo final de la Licenciatura en Nutrición (LN), de la Facultad de Bioquímica y Ciencias Biológicas (FBCB) de la Universidad Nacional del Litoral (UNL).

Este entramado reflexivo y crítico se realizó a partir de las voces de los y las extensionistas en sus diferentes roles, y continuó a través del proceso de investigación con referentes claves de cambio, desde lo individual y lo colectivo.

La dinámica propuesta de PEIS mediante la educación alimentaria nutricional (EAN) resultó esencial para trabajar con el nivel inicial y sus diversas problemáticas nutricionales. Lo vivenciado por los actores posibilitó la flexibilización de miradas y pensamientos. Como postula Camilloni: "Cuando el aprendizaje es experiencial, quien aprende lo hace en las condiciones sociales de la utilización auténtica de los conocimientos" (2013, p. 17).

Se presentan entonces las reflexiones en cada vínculo de este tejido por medio de sus voces, y se analizan críticamente aquellos obstáculos que fueron detectados a través del diálogo conjunto, para re-transformar la propuesta como proyecto, convirtiéndose en una herramienta superadora y de avance.

Hacia el final del trabajo, se comparte la voz de las personas extensionistas investigadoras, cuyas intervenciones en el campo y el proceso científico permitieron encontrar otras preguntas, enfrentando una realidad que interpela la formación profesional. Así, la extensión universitaria vuelve a ser el punto de partida para repensar las propias prácticas.

Al hablar de tejer redes, cobra protagonismo cada extensionista participante del PEIS a lo largo de tres años. En ese entramado, distintas instituciones de la ciudad de Santa Fe y alrededores desarrollaron actividades y encuentros enriquecedores desde abril de 2017 hasta diciembre de 2019. Se citan entonces el Jardín de Infantes N. ${ }^{\circ} 68$ "Dr. Camilo Corti" y sus anexos (Escuela N. • 569 "República del Paraguay" y Escuela N. 21 "Pedro de Vega"); los Jardines Municipales Coronel Dorrego y Pro Mejoras Barranquitas; las salas de nivel inicial de la Escuela Primaria N. ' 1065 "Manuel Belgrano" de Ángel Gallardo (Monte Vera) y Jardín N. ${ }^{\circ} 130$ "Jorge Baialardo" de San José del Rincón. 
Revista Universidad en Diálogo • Vol. 11, N. ${ }^{\circ}$ 1, Enero-Junio, 2021 • 157-178

ISSN 2215-2849 • EISSN: 2215-4752

URL: http://www.revistas.una.ac.cr/index.php/dialogo/index CoRReo eleCtrónico: universidadendialogo@una.cr DOI: http://doi.org/10.15359/udre.11-1.6

En ese proceso participaron más de 892 niños y niñas, de 1 a 3 años (jardines municipales), y de 3 a 5 años (jardines provinciales). Como beneficiarias indirectas en algunos casos, y directas en otros, las familias también estuvieron presentes; además de 52 docentes y personal directivo. Por último, participaron 40 estudiantes de la LN y de la Licenciatura en Diseño de la Comunicación Visual (LD) de la Facultad de Arquitectura, Diseño y Urbanismo (FADU), quienes compartieron y llevaron adelante en forma conjunta 45 propuestas didácticas.

Recuperar las voces permitió evaluar con una mirada real y crítica las intervenciones realizadas, así como la propuesta teórica, contrastando con la realidad. Además, la utilización de varios instrumentos y documentos (triangulación metodológica) permitió la colecta de datos y vivencias, para la reflexión grupal y el empoderamiento del proceso de extensión.

\section{EI camino previo recorrido del PEIS}

Los inicios del PEIS se remontan al año 2014 con Prácticas de Extensión en Educación Experiencial (PEEE) en el nivel inicial, cuyo propósito consistió en la promoción de alimentación sana y segura en jardines municipales y privados de la ciudad de Santa Fe. Durante la propuesta, se desarrollaron talleres, actividades, juegos y la inclusión de piezas con diversos lenguajes comunicacionales artísticos expresivos, destinados a la comunidad en general. Se definieron en ese entonces como fortalezas: la aproximación de la comunidad universitaria a los niños y las niñas y sus familias; la construcción de recursos didácticos para compartir e interactuar con los mensajes de alimentación saludable y la búsqueda de actividades y contenidos acordes a la edad. Esto se reconoció como un trabajo arduo pero enriquecedor, que evidenció el compromiso y la actitud profesional de los y las extensionistas (Ravelli, Paniccia y Figueroa, 2016). Sin embargo, se marca como debilidad la ausencia de sistematización de información y datos a través de propuestas de investigación, que posibiliten una integración con la función extensionista.

Por lo expuesto es que posteriormente las PEEE, a través de la práctica profesional (PP) de LN, comienzan a ser llevadas a diversos jardines municipales, para diseminar las propuestas de EAN a través de diversos dispositivos lúdico-pedagógicos (DLP). Así es que, en ese recorrido, las PEEE mutan hacia el desarrollo de un PEIS, continuando hasta la actualidad.

En el trayecto, se realizaron $-\mathrm{y}$ continúan desarrollándose- diversos trabajos de investigación, los cuales son socializados en espacios académicos, presentando resultados, recursos lúdicos e integrando trabajos finales de la 
LN. Según Boimvaser, Ravelli, Gioria y Gornati, "estas dos experiencias resultaron no solo una oportunidad para la proyección del título de grado de las tesistas sino una posibilidad de integración de acciones conjuntas (tríada extensión, docencia e investigación) que potenciaron y resignificaron los objetivos propuestos" (2018, p. 254).

Como se postula desde la Secretaría de Extensión Social y Cultural de la UNL, los PEIS son:

Proyectos orientados al abordaje de la problemática socioeconómica, socio ambiental y/o socio cultural en forma interdisciplinaria y conjunta con las organizaciones destinatarias del medio, y que su finalidad, es contribuir a la promoción de la expansión de las capacidades humanas de la población objetivo, con una meta clara en relación con el mejoramiento de la calidad de vida y asegurando el impacto de sus acciones. (Plataforma Virtual UNL, 2020).

El desarrollo de la tarea extensionista debe acompañarse con la retroalimentación continua del proceso. En esa dirección, es que se propuso como grupo de extensionistas investigadoras evaluar el impacto del PEIS, con el fin de sistematizar un registro del proyecto, resignificando las diferentes voces involucradas en el mismo. Esta evaluación resulta un antecedente formal dentro de los proyectos de extensión que involucran a la EAN en el nivel inicial.

Socializar las diferentes miradas de quienes participan en el proyecto y reforzar la necesidad de una instancia de evaluación para tejer redes y vínculos con la comunidad favorece no solo la evaluación de los objetivos planteados, sino también la retroalimentación continua del PEIS.

\section{La diversidad de actores en el PEIS y su relación con la educación alimen- taria nutricional en el nivel inicial}

El proyecto entrelazó las acciones y voluntades de diversos actores, cuyo propósito fue aportar y acompañar conocimientos, saberes y actitudes en relación con la EAN en edades tempranas. Según Ravelli et al., "se debe poner énfasis, especialmente en el nivel educativo inicial, y en todos los actores que en ella actúan; niños, maestros, la familia y la comunidad en general" (2016, p. 258).

En este apartado, entonces, se describe a los actores participantes y su relación con el proceso realizado. 
Las primeras personas protagonistas fueron los niños y las niñas del nivel inicial, sujetos cuyas características favorecen la construcción de valores y solidaridades y son permeables ante lo novedoso y distinto. Son personas potentes, energéticas y con capacidad de autoconstrucción, mientras transforman el mundo, con la fuerza de quien crece y desea crecer, con la riqueza de la curiosidad, del asombro que las lleva a preguntarse el porqué de cada cosa, en la plenitud de sus sentidos, originales, creativas, con el deseo de aprender y de vivir (Ministerio de Educación de la Provincia de Santa Fe, 2020).

Los niños y las niñas son el resultado de una historia vivida. En esa línea, López Sánchez (2010) afirma que cada persona tiene cualidades innatas favorables (creatividad, bondad, emociones, entre otras); desde antes del nacimiento se generan condiciones que incidirán en un futuro, implicando el ambiente y el entorno. Por eso, la presencia de la persona adulta en la vida de los niños y las niñas es clave, ya que debe brindarles las herramientas y condiciones para transformar estas cualidades y utilizarlas según la ética, para resolver conflictos desde la cooperación y la ayuda.

Como afirman Boimvaser et al. (2018):

Se sostiene la importancia de las intervenciones dirigidas a la población infantil de edad escolar, ya que los hábitos alimenticios de niños y niñas se forman desde edades muy tempranas y se van delineando a través de su vida, creando un patrón de conductas en la edad adulta. (p. 255)

Las familias de los niños y las niñas, a través de acciones directas (encuentros) e indirectas (mediante lo comunicado por sus hijos e hijas), resultaron claves en el sostenimiento de los hábitos saludables acordados con el equipo extensionista. El desarrollo de la EAN posibilita "que las familias reconozcan el derecho a la alimentación, y en consecuencia puedan seleccionar los alimentos necesarios para desarrollarse y funcionar en condiciones óptimas de salud" (Cossani et al., 2011, p. 2).

Por otra parte, las personas docentes - tanto del nivel inicial como del universitario- también fueron actores claves en el proyecto, ya que sostuvieron, por un lado, los roles tradicionales de planificar, evaluar y mediar el proceso de aprendizaje en relación con la EAN, pero también pudieron diseñar y propiciar las situaciones en las que el niño y la niña se involucraron de manera activa y constructiva.

La EAN posee un cuerpo de conocimientos básicos que se puede matizar y adaptar a los intereses de cada institución o nivel; y todo lo relacionado 
URL: http://www.revistas.una.ac.cr/index.php/dialogo/index

CORREO ELECTRÓNICO: universidadendialogo@una.cr

DOI: http://doi.org/10.15359/udre.11-1.6

a alimentación y nutrición tiene un amplio componente educativo desde su origen (Vega, Manzanera y Armenta, 2013).

Se observó entonces, como hecho favorecedor, que las personas docentes de nivel inicial conocían y manejaban aspectos sobre el desarrollo evolutivo de la población infantil, sobre las formas en las que aprenden, sus intereses y necesidades básicas, sus derechos, potencialidades y su realidad sociocultural; mientras que la persona docente universitaria extensionista analizó la demanda de las instituciones y seleccionó estrategias y recomendaciones, para desarrollar interdisciplinariamente las acciones y lograr las metas propuestas.

Las y los docentes, de nivel inicial y universitario, como personas mediadoras de la tarea educativa, propician nuevos aprendizajes, creando condiciones para que los niños y las niñas descubran, exploren, manipulen e indaguen a través de diversas acciones y actividades de EAN, lo que genera además que puedan relacionar los nuevos aprendizajes con sus conocimientos previos.

En esta línea, fue responsabilidad del equipo extensionista organizar, junto con los y las docentes del nivel, diversidad de ambientes para desarrollar un clima favorecedor de los derechos y de los aprendizajes, para promover la libertad de acción y respeto y para generar oportunidades e interacciones con elementos del entorno. Esto les posibilitó a los niños y las niñas conocer y vivenciar juegos y actividades de alimentación, nutrición y salud.

Por último, parte de los y las estudiantes practicantes de LN y LD (FADU) participaron en todo el proceso propuesto en el PEIS. Se involucraron activamente desde la planificación inicial de las actividades y reuniones previas con docentes y personal directivo hasta la creación de dispositivos lúdicos y su implementación en talleres y jornadas de EAN con niños y niñas. También se contó con la participación de estudiantes a partir del tercer año de la carrera, bajo la modalidad de un voluntariado extensionista; esto posibilitó la inclusión temprana en situaciones y problemáticas propias asociadas al ejercicio profesional.

De acuerdo con Ordoñana (2003), la labor de la persona profesional no se ciñe exclusivamente en la transmisión de una serie de conocimientos sobre el proceso salud-enfermedad a la comunidad, por el contrario, el desarrollo de programas y actividades de educación para la salud contempla multitud de objetivos intermedios, entre los que se encuentran cuestiones tan complejas como formar, capacitar, motivar e incidir sobre las actitudes y los valores, para finalmente provocar cambios que apunten al desarrollo social. 


\section{Proceso de evaluación del trayecto recorrido}

En el trayecto recorrido en el marco del PEIS, tal como se mencionó anteriormente, se integró un trabajo final de grado de LN, con el fin de evaluar el impacto de las acciones desarrolladas, resignificando las voces y miradas de las personas participantes involucradas en el proyecto, para considerar una noción más amplia de impacto que incluye el medio ambiente y la comunidad.

En la actualidad, el concepto de impacto social incorpora no solo los resultados previstos, sino también aquellos que no se previeron. De acuerdo con Libera Bonilla, "se contemplan los efectos, tanto positivos como negativos que se pudieran presentar luego de la implementación de un determinado programa o proyecto en un grupo social o una comunidad" (2007, p. 4).

Para el proceso de evaluación, se llevó adelante un estudio de tipo cualicuantitativo, descriptivo. El mismo constó de varias etapas, con la utilización de diferentes técnicas e instrumentos para la recolección de datos.

En primera instancia, se determinaron los aspectos estructurales y contextuales más relevantes del PEIS. Como parte de la investigación, se procedió a analizar el proyecto educativo institucional (PEI) de cada institución educativa, con el objetivo de encontrar puntos en común entre las problemáticas alimentarias detectadas por los establecimientos y los objetivos de EAN que persigue el PEIS. La metodología empleada fue el análisis documental y la lectura de informes previos.

Asimismo, uno de los objetivos de la investigación consistió en describir las estrategias metodológicas y los recursos utilizados en el marco del PEIS, para lo cual se efectuó una revisión de informes de PP de LN; análisis de registros de planificaciones desarrolladas por el equipo extensionista; se procedió a la lectura de artículos y publicaciones elaborados en el contexto del PEIS, así como también a la búsqueda de registros fotográficos, audiovisuales y recursos didácticos construidos en este marco.

En la etapa final del trabajo, se recabaron y analizaron las voces de los miembros de las instituciones de nivel inicial y de los y las participantes de la LN-FBCB, para conocer sus percepciones sobre los efectos alcanzados por el proyecto. Para ello, se entrevistó, mediante grupos focales, a docentes y personal directivo de los establecimientos educativos y a las familias de los niños y las niñas, así como también a personas estudiantes voluntarias y practicantes de LN, con el fin de sumar su valoración acerca de su paso por el 
URL: http://www.revistas.una.ac.cr/index.php/dialogo/index

CORREO ELECTRÓNICO: universidadendialogo@una.cr

DOI: http://doi.org/10.15359/udre.11-1.6

PEIS en la formación como profesionales. Se utilizó la entrevista por medio de grupos focales, que, como enfatiza Mayan (2001):

Es una técnica que permite recolectar gran cantidad de datos sobre un área particular de interés dentro de un corto periodo de tiempo, observar la interacción entre los/as participantes, la cual es una parte integral del significado de los datos, y aprender no solo sobre las opiniones y actitudes de los participantes sino también sobre sus experiencias y perspectivas. (p. 19)

Finalmente, se desarrollaron talleres para resignificar saberes y recuperar contenidos junto a los niños y las niñas que participaron en las actividades propuestas por el PEIS. Para el registro de la información proveniente de estos talleres se llevó adelante la observación participativa, la cual, como propone Muñoz Razo (1998), resultó apropiada, ya que permitió conocer más de cerca las características, la conducta y el desenvolvimiento del fenómeno en su medio ambiente, comprobando modificaciones y variaciones predeterminadas para observar los posibles cambios de comportamiento.

Tal como se percibe, se emplearon diversidad de métodos para analizar el impacto de la propuesta y para explicar una realidad concreta. La triangulación de datos ayudó a obtener un mayor desarrollo y enriquecimiento teórico, conformándose en la búsqueda de fuentes de datos según criterios espaciotemporales y distintos niveles de análisis, según la persona y el objeto de estudio (García, García, González, Carvalho y Catarreira, 2016).

\section{La experiencia extensionista: resultados alcanzados}

En el proceso de evaluación del impacto del PEIS realizado por las extensionistas investigadoras se llevaron a cabo diversidad de talleres junto a noventa y ocho niños y niñas; se logró entrevistar a ochenta y tres representantes de familias; y se pudo recabar información de veintisiete docentes y directivos, y de treinta y cinco estudiantes de la LN. Sobre estos datos, los cuales se obtienen de un recorte de toda la población implicada en los tres años del PEIS, se puede interpretar que el mismo logró un gran alcance a nivel social y territorial.

Si bien el relevamiento de datos cuantitativos aporta certezas sobre el aumento en el consumo de frutas y verduras y una fuerte apreciación sobre la importancia de mejorar hábitos de higiene, para este trabajo fue necesario "mirar desde otro lado" y comprender contextos estructurales y situacionales, por lo que la multiplicidad de instrumentos y técnicas en la recolección de información 
y la narración de fenómenos conjugan diversidad de métodos que priorizan el análisis del contexto, la historia, las relaciones, las representaciones y la participación social (Minayo, Assis y Souza, 2008).

\section{Resultados sobre el entramado PEI-PEIS}

Como se manifestó, en primer lugar, se trató de vincular el PEI con el PEIS. El primero, como principio ordenador de la institución, establece como prioridad que el cambio social y participativo requiere decisiones contextualizadas de acuerdo con la dinámica y la realidad del entorno (Barrientos, 2020); mientras que el segundo plantea que las organizaciones e instituciones involucradas deberán participar activamente en todas las instancias del proyecto, es decir, desde su formulación, desarrollo, monitoreo y evaluación de impacto (UNL, 2020).

Se vislumbra entonces, dentro de los factores convergentes, la coherencia en los objetivos que persigue cada uno de los proyectos. En este sentido, en el análisis documental se pudieron observar algunos criterios en común, los cuales se presentan a continuación:

\section{Objetivos de los PEI:}

"Desarrollar hábitos, para favorecer su crecimiento sano y armónico" (PEI Jardín N. ${ }^{\circ} 68$ Camilo Corti, 2017).

"Conocer los cuidados alimenticios que requiere su cuerpo para lograr un crecimiento sano y actividad física apropiada" (PEI Escuela N. ${ }^{\circ}$ 1065 Ángel Gallardo, 2019).

"Cuidado de la salud y trabajo con otras instituciones" (PEI Jardín N. ${ }^{\circ}$ 130, Rincón, 2019).

\section{Objetivos generales del PEIS:}

"Promover el consumo de frutas y verduras en niños y niñas que asisten a los Jardines Municipales y Provinciales seleccionados".

"Fomentar el cuidado de la higiene personal y de los alimentos en los niños y las niñas". 
Se puede observar que los objetivos generales del PEIS concuerdan con los propósitos de los PEI. Es decir que las instituciones pudieron vincular sus metas con el PEIS, asumiendo el compromiso de trabajar la temática sobre alimentación saludable e higiene personal y de los alimentos como eje central durante el periodo lectivo, con la posibilidad de involucrar todas las áreas (ciencias, dibujo, lectura, educación física), además de otros proyectos con los que trabaja cada institución.

En este recorrido se pudo acompañar en la construcción y el fortalecimiento de los hábitos alimentarios a través de la EAN, abordando problemáticas comunes que afectan a la población infantil que asiste a estos jardines. Los PEI cobijan actividades, intervenciones y contenidos a trabajar desde la sala a lo largo del año, y que el PEIS fortaleció desde lo académico, disciplinar y específico, pero sobre todo desde lo vincular y actitudinal. Esta dinámica de trabajo permitió lograr resultados prometedores en pos de mejorar la calidad de vida de la comunidad.

En esta línea, para ilustrar lo reflexionado, citaremos respuestas del personal directivo y de docentes de los establecimientos educativos:

Desde arte se trabajó con Arcimboldo, con sus imágenes exóticas de frutas y verduras en la cabeza, con ciencias donde las chicas sacaron los pigmentos, se trabajó sobre la descomposición, un montón de cosas que a nosotros nos sirvió para trabajar en las distintas áreas la transversalidad. (Directora 1, Jardín N. ${ }^{\circ}$ 68, Santa Fe, 2019)

A partir de las intervenciones del PEIS el comedor escolar comenzó a ofrecer de merienda o postre frutas, y las docentes planifican actividades como el armado de una huerta, recetas con frutas, charlas con otros profesionales de salud. (Directora 2, Escuela N. ${ }^{\circ} 1065$, Ángel Gallardo, 2019).

Desde la copa de leche comenzamos a regular el consumo de azúcar y golosinas. Además de sugerir a las familias que en lugar de comprar alfajores en el kiosco a la salida del jardín, compren una fruta. (Directora 3, Jardín N. 130, Rincón, 2019)

El proyecto "Cuidando nuestro cuerpo seremos saludables y fuertes" podíamos trabajarlo en paralelo al PEIS, al igual que el proyecto de Feria de Ciencias. (Docente 1, Salas 4 y 5, Jardín N. ํ 130, Rincón, 2019). 
Es importante incorporar este tipo de actividades porque generan cambios incluso institucionalmente, por ejemplo para el picnic de la primavera se transformó, se prepararon licuados, jugos, otras preparaciones. (Docente 2, Jardín N. ${ }^{\circ} 130$, Rincón, 2019)

Estas acciones marcan, tal como expresa Rafaghelli (2013), el compromiso de trabajo que asumen las instituciones, entendiéndose como "procesos factibles por y en las prácticas colectivas, en la escucha, el diálogo y la negociación permanente de significados entre los actores involucrados" (p. 23).

\section{Resultados sobre el tejido de las voces infantiles-PEIS}

Con el objetivo de registrar las voces de los niños y las niñas y retomar el trabajo a lo largo del PEIS, se llevaron adelante talleres y, a través del registro observacional, se relevaron sus respuestas con el fin de evaluar la identificación de frutas y verduras. La estrategia didáctica "A degustar" permitió también observar las reacciones y emociones de los niños y las niñas al momento de probar.

Además de reconocerlas y mencionar aquellas frutas que podían consumir con cáscaras o no, las formas de consumirlas en el hogar, el conocimiento sobre sus semillas, siembra y obtención de alimentos, el recurso provocó un llamado de atención y una excelente aceptación.

Las extensionistas investigadoras acompañaron generando preguntas sobre características de las frutas y verduras presentadas en la actividad. Estos son algunos de los comentarios que surgieron:

"Las frutillas tienen las semillas por fuera" (Niños y niñas, Sala 5 del Jardín N. ${ }^{\circ} 68$ y Escuela N. $\left.{ }^{\circ} 1065,2019\right)$.

"Las bananas tienen las semillas en el centro" (Niños y niñas, Sala 5 del Jardín N. ${ }^{\circ} 68$ y Escuela N. ${ }^{\circ} 1065,2019$ ).

Al momento de la degustación:

"Me gusta mucho la banana, la como en casa siempre" (Niña, Sala 5, Jardín N. ${ }^{\circ} 130,2019$ ).

"El limón tiene la piel suave igual que la naranja" (Niño, Sala 4, Jardín N. $\left.{ }^{\circ} 130,2019\right)$.

"El tomate lo como con la lechuga" (Niña, Sala 4, Escuela N. $\left.{ }^{\circ} 1065,2019\right)$. 
A través de la experimentación y degustación con preparaciones y recursos didácticos concretos de alimentación, se observó el interés y la motivación de los niños y las niñas hacia la alimentación saludable, además reconocieron las fases previas como la selección y preparación de alimentos.

Los diversos programas de educación sensorial llevados a cabo en el ámbito escolar han mostrado resultados positivos, tanto en la reducción de la neofobia alimentaria como en el aumento de la disposición a probar alimentos desconocidos.

"La educación sensorial, aumentando la conciencia y la curiosidad hacia los alimentos y mediante la interacción con los iguales, estimula a los niños a probar alimentos desconocidos" (Maíz, Urdaneta, Allirot, 2018, p. 137).

\section{Voces de las familias: fortaleciendo la trama niños/niñas-PEIS}

Los espacios compartidos directamente con las familias se produjeron durante algunos eventos, gestionando y generando talleres, juegos y degustación de preparaciones. Además, de manera indirecta, se comunicó semanalmente a través de notas, adivinanzas, poemas o dibujos lo que se trabajó con los niños y las niñas en relación con buenos hábitos.

En los grupos focales en general hubo un marcado aumento del consumo de frutas y verduras en los niños y las niñas que participaron en los talleres. Además, se evidenció entusiasmo en las madres y los padres, ya que sus hijos e hijas, luego de las actividades, pedían alimentos probados y preparaciones frutales (trufas, ensaladas, licuados), así como hamburguesas de legumbres.

Otro relato familiar tiene que ver con que reconocían la importancia de evitar jugos, gaseosas y golosinas. Esto fue visible porque reclamaban o insistían en comer frutas y tomar agua. Resaltaron además el interés de los niños y las niñas en realizar con toda la familia el lavado de manos varias veces al día y lavarse los dientes luego de cada comida.

Algunas de las expresiones textuales de las familias acompañan las conclusiones obtenidas:

"Mi hijo me contaba: esto tengo que comer para crecer fuerte y sano" (Mamá 1 de niño de Sala 5, Jardín N. ${ }^{\circ}$ 68, Santa Fe).

"Mi hija al llegar a casa me empezó a pedir la manzana entera, bien lavada y que no le saque la cáscara porque hacía bien" (Mamá 2 de niña de Sala 5, Escuela N. ${ }^{\circ} 1065$, Ángel Gallardo). 
"Mi hijo pedía que le cocine porotos, y yo la verdad que no sabía ni cómo prepararlos" (Mamá 3 de niño de Sala 5, Jardín N. ${ }^{\circ}$ 130, Rincón).

Los lazos estrechados con el PEIS se manifiestan a través del reconocimiento del trabajo con los niños y las niñas, de la aceptación de los alimentos compartidos en los encuentros, recordando costumbres familiares y resignificando sus tradiciones. En las jornadas, efemérides y reuniones de padres y madres pudieron acompañar aportando sus realidades y vivencias. Se observó en casi todas las familias el interés por las actividades realizadas, tendientes a seguir con las acciones propuestas por el equipo extensionista.

Otras frases de las familias detectaron la repercusión del PEIS en los niños y las niñas:

"Mi hijo al llegar a casa me contó que habían hecho torta con frutas y que estaba rica" (Mamá 4, Sala 5, Jardín N. ${ }^{\circ}$ 68, Santa Fe).

"Mi hijo volvía muy entusiasmado luego de los talleres, y me pedía que le prepare ensaladas frutales" (Mamá 5 de niño de Sala de 5, Escuela N. ${ }^{\circ} 1065$, Ángel Gallardo).

"Mi hija al llegar del Jardín, me contaba lo que habían aprendido de las lentejas" (Mamá 6 de niña de Sala de 5, Jardín N. ${ }^{\circ}$ 130, Rincón).

Para la población estudiantil y las personas docentes universitarias conocer el entramado familiar aportó desde lo social y cultural, y posibilitó comprender ciertas preferencias alimentarias, así como las actitudes y los comportamientos de los niños y las niñas. Esto permitió explicar algunos resultados de las intervenciones, las cuales son multifactoriales. Comprender esta complejidad es importante al momento de la planificación para asegurar el logro de una intervención alimentaria. "El contexto de la actividad situada cultural e históricamente es crucial para potenciar la formación de los universitarios, y así diseñar recursos y programas más eficientes" (Rafaghelli, 2013, p. 26).

\section{Voces de docentes y personal directivo de los establecimientos; vo- ces de docentes y estudiantes $\mathrm{LN}$}

Se establecieron numerosos canales de comunicación para generar propuestas adecuadas e innovadoras en las tres comunidades donde se trabajó. Por un lado, se realizaron reuniones periódicas, cuyo fin era acercar inquietudes, actividades y dudas que, a través de las experiencias de unas y las voluntades de otras, podían resolverse. 
URL: http://www.revistas.una.ac.cr/index.php/dialogo/index

CORREO ELECTRÓNICO: universidadendialogo@una.cr

DOI: http://doi.org/10.15359/udre.11-1.6

En este sentido, la experiencia en docencia y en aspectos didácticos significó para la población estudiantil voluntaria y para los y las practicantes extensionistas un apoyo esencial para la implementación de talleres y actividades.

Se logró sistematizar el trayecto recorrido a través de planificaciones semanales consensuadas entre personas docentes de jardines y personas docentes voluntarias y practicantes de la $\mathrm{LN}$, lo que generó un ambiente propicio de enseñanza y aprendizaje para los niños y las niñas y permitió el desarrollo de contenidos y actividades de manera óptima, porque toda la comunidad educativa se sumó al fin establecido: cambio de hábitos, mayor consumo de frutas y verduras, hidratación, mejora de la higiene personal y de los alimentos, entre otros aspectos.

Los grupos focales realizados con docentes y personal directivo en las diferentes instituciones resaltaron el trabajo realizado por la población estudiantil extensionista, destacando que los tiempos y espacios para desarrollar cada actividad eran óptimos según la dinámica escolar, además, sin reemplazar el rol docente, se fortaleció y se aprendió de él.

Es necesario reconocer aquí que la persona practicante o voluntaria de LN no posee específicamente una preparación pedagógica, lo cual puede resultar una dificultad en el desempeño de las actividades; no obstante, el acompañamiento docente es clave para la retroalimentación continua. Así, reivindicamos lo dicho por Menéndez et al., "se pueden crear nuevos conocimientos interviniendo en la práctica, en la actividad misma, de allí la necesidad de participar en escenarios sociales amplios" (2013, p. 89).

Como se mencionó, las propuestas y los recursos didácticos fueron creativos e innovadores, lo cual resultó esencial para captar la atención de los niños y las niñas y para desarrollar los contenidos programados. Esta particularidad fue mencionada por los y las docentes en varias oportunidades, ya que notaban cambios en la población infantil en relación con sus acciones cotidianas vinculadas con su alimentación e higiene.

En relación con lo anterior, de las entrevistas llevadas a cabo con los y las docentes, se destacan las siguientes expresiones:

"Para mi eran muy creativos, y creo que fueron muy lúdicos para ellos, el de la Vaca fue fantástico" (Docente 3, Sala 5, Jardín N. ${ }^{\circ}$ 68, Santa Fe, 2019).

"Los contenidos fueron claros, bien trabajados, hubo bastante material didáctico desplegado, que llamaron el interés de los chicos" (Docente 4, Sala 5, Escuela N. ${ }^{\circ} 1065$, Ángel Gallardo, 2019). 
Las intervenciones fueron positivas, pero resultó un impedimento, por momentos, la cantidad de niños y niñas, se notaba que a veces los recursos no alcanzaban para todos, se distraían, daban vueltas por el SUM, los niños y niñas en sala de 4 al principio todavía no estaban acostumbrados a esas dinámicas. (Docente 5, Sala 4, Jardín N. ${ }^{\circ}$ 130, Rincón, 2019).

Por otra parte, en las entrevistas los y las docentes realizaron aportes para mejorar las acciones por parte de las personas voluntarias extensionistas. Un aspecto sustancial que se observó en sus respuestas fue que es necesario conocer con mayor profundidad, previamente, la comunidad y la dinámica escolar, lo cual permitiría mejoras en las planificaciones y en el vínculo establecido con la institución.

Lo expuesto anteriormente fue considerado por el equipo extensionista como un desafío a atender y proyectar a futuro en el marco del PEIS, entendiendo que la tarea extensionista está en permanente proceso de construcción y deconstrucción junto a los actores sociales. En esta línea, "el propósito de estos proyectos, es que quienes participan en el mismo compartan y construyan socialmente conocimientos que impliquen un mejoramiento sustantivo en el contexto específico de su propia cultura" (Rafaghelli, 2013, p. 32).

\section{Voces de las personas estudiantes extensionistas LN: entretejiendo saberes con el PEIS}

Por parte de los y las estudiantes de LN, se evidenció la importancia otorgada a la realización del PEIS y el desarrollo de actividades de EAN en el ámbito escolar, dado que es el momento donde comienzan a adoptar hábitos alimentarios.

Sus entrevistas remarcaron como fortaleza la flexibilidad y la dinámica del trabajo en el territorio, ya que las actividades y propuestas podían ser mejoradas, repensadas y reformuladas en caso de presentarse dificultades. Las personas extensionistas podían administrar nuevamente los contenidos, el vocabulario, la organización del tiempo y la dinámica en función de las observaciones y sugerencias realizadas oportunamente por docentes y personal directivo de los establecimientos.

Asimismo, resultó valioso tener instancias previas con docentes que ayudaron a vincularse con el grupo y a tener una mejor preparación. Esto fortaleció el intercambio entre estudiantes de $\mathrm{LN}$ y docentes. 
Sus voces reflejan el arduo trabajo de extensión en el territorio, donde nada es rígido, permitiendo la construcción del saber. Tal como lo expresa Camilloni, sobre el aprendizaje experiencial, "la construcción de conocimientos estratégicos, en situaciones reales, que luego sabrán cómo transferir a situaciones similares, son experiencias educativas en tanto y en cuanto promueven procesos reflexivos, reconocimiento de analogías, contradicciones y análisis, en pos de favorecer la comprensión” (2017, p. 9).

Por otro lado, hubo factores externos que en algunas ocasiones debilitaron el proceso iniciado; actividades improvisadas o extras de la institución, paro docente, actos, feriados, días de lluvia, entre otros. Eso implicó reorganización de cronograma, en algunos establecimientos reducción de encuentros y reflexionar como extensionistas sobre la realidad cotidiana fuera de las estructuras teóricas, y que en determinadas circunstancias escapa a la voluntad de los actores. Estas situaciones se plantearon en espacios destinados a resolver las dificultades mencionadas (encuentros previos a las actividades entre docentes-estudiante, reuniones plenarias, clases de consulta, encuentros bimestrales del PEIS).

Para la mayoría de estudiantes extensionistas resultó un desafío el trabajo con la población infantil. Esto podría deberse a la escasez de estudios relacionados a EAN en el nivel inicial, recursos didácticos limitados, falta de áreas de formación didáctica-pedagógica en la carrera de LN que incluyan las herramientas para la resolución de las debilidades planteadas.

Aun así, a medida que transcurrió el trayecto, se logró un mejor manejo de grupos y la identificación rápida de sus necesidades y limitaciones. En este punto, la preparación y el empleo de recursos didácticos adecuados, el diálogo constante con docentes y con el equipo extensionista y el acompañamiento de personas becarias y otras voluntarias posibilitaron el avance hacia otros retos.

Al reflexionar sobre lo transitado, se revalorizó el permanente feedback entre la universidad pública y la comunidad, no pensado como dos bloques independientes y rígidos, sino como un conjunto que se retroalimenta, siendo clave para la formación profesional.

Como menciona Menéndez et al., "es preciso ver a los actores interactuando con la realidad, y es en esa relación dialéctica con la misma que concebimos un proceso de constante transformación y construcción, donde la relación universidadsociedad sea promotora de acciones transformadoras" (2013, p. 50). 
Algunos fragmentos de respuestas de las personas estudiantes extensionistas permiten ilustrar lo anterior:

"Aprendí a trabajar en equipo, disfrutando de cada etapa, a entender los tiempos de los niños y a disfrutar de su aprendizaje. También a entablar una relación con el personal de las instituciones en las que trabajamos" (practicante 1, LN, 2017).

"Trabajar con niños pequeños que enseguida te transmiten alegría y es increíble cuando demuestran interés por las propuestas que les llevábamos y más aún cuando veíamos que iban incorporando los contenidos de los talleres" (voluntario 2, LN, 2018).

"Participar del PEIS no solo influyó en mi formación profesional, sino en mi orientación. Porque ahora no se si la quiero ejercer de la misma forma que pensaba antes" (practicante $3, \mathrm{LN}, 2019$ ).

\section{Reflexiones finales y proyecciones}

Este trabajo posibilitó identificar los objetivos y contenidos comunes abordados en los PEI y PEIS, complementando ambos los aspectos institucional, social y pedagógico, factores claves para el desarrollo de la función extensionista. En este sentido, distinguir objetivos comunes, delimitar competencias específicas de los sectores sociales implicados y planificar las acciones integradas es entender la promoción de la salud desde un enfoque integral (Parrilla, MuñozCadavid y Sierra, 2013).

Las voces de los actores implicados acuerdan sobre la necesidad de abordar la EAN desde edades tempranas de una manera integral, atendiendo al contexto y a la realidad atravesada.

En el caso de los niños, las niñas y sus familias, se destacó la identificación, la exploración y el reconocimiento de alimentos poco conocidos, además de la apropiación de saberes y haceres necesarios para la modificación y/o adopción de hábitos saludables, tales como la higiene de manos y el cepillado de dientes.

La promoción de hábitos y calidad de vida debe centrarse no solo en el entorno escolar, sino también en la familia. Es fundamental la alfabetización y participación familiar dentro de enfoques que faciliten modelos alimentarios saludables (Gillespie y Johnson-Askew, 2009). 
Esta propuesta pudo atesorar voces y puntos de encuentro desde un enfoque comunitario, adoptando iniciativas emergentes y complejas que retroalimentaron la participación de los actores y la misma EAN, como herramienta promotora de salud.

Las voces de los y las docentes y del personal directivo, por otra parte, dieron cuenta del trabajo mancomunado con personas extensionistas universitarias, pudiendo vincular los objetivos institucionales con el PEIS, lo que sirvió de plataforma para el compromiso de todas las personas. Esto se manifestó en el interés unánime de seguir trabajando los próximos años.

Una de las fortalezas mencionadas por el grupo docenteen relación con estudiantes y personas voluntarias es la capacidad de elaborar e implementar actividades lúdicas creativas que tuvieron repercusión en los niños y las niñas. Además, a través del diálogo docente-estudiante extensionista, la retroalimentación constante permitió modificar y adecuar las propuestas didácticas.

Respecto a lo valorado por los y las estudiantes y personas voluntarias, se destacó la extensión como proceso transformador. Por un lado, la posibilidad de atravesar los muros academicistas para acompañar a la comunidad educativa con mensajes de alimentación, nutrición y calidad de vida; por el otro, aprender de la población infantil, su familia y la comunidad educativa.

Cómo tejer vínculos, cómo desenvolverse en una realidad que no es la propia y cómo utilizar lenguajes culturales diversos les permitió enriquecerse humana y profesionalmente, aportando una mirada crítica y comprometida con la realidad y con su futura profesión. En este sentido, si bien hubo limitaciones y dificultades, estas trataron de ser resueltas a través del diálogo y de la reflexión frecuente entre los actores implicados, en los espacios destinados para tal fin.

A modo de reflexión final, la evaluación del impacto del PEIS, ponderando las voces de sus actores, no se considera un cierre, sino un nuevo punto de partida. Repensar la extensión, a través del hilo conductor de la EAN, entretejiendo saberes y voluntades, resignifica el rol del LN y el compromiso asumido de los actores participantes.

Muchas preguntas quedan abiertas, quizás sean otras las voces que podrán responderlas y continuar la labor extensionista comenzada: 
¿De qué manera se puede mejorar lo realizado? ¿Es posible potenciar y multiplicar las acciones desarrolladas? ¿Qué otros actores podrían comprometerse? ¿Es posible seguir evaluando el impacto? ¿De qué forma? ¿Cómo se mira el PEIS a partir de esta evaluación? ¿Se puede repensar el rol profesional desde, en y para la extensión?

Estas interrogantes y muchas otras, que pueden surgir a lo largo del proyecto, son el inicio de futuras acciones, seguramente superadoras, que continúe desarrollando la EAN en el nivel inicial.

\section{Referencias}

Barrientos, B. R. (2020). Qué es un proyecto educativo institucional. http:// www.atmos.cl/inicio $1 /$ book/export $/ \mathrm{html} / 18$

Boimvaser, N., Ravelli, S., Gioria, E. y Gornati, F. (2018). Extensión, docencia e investigación como estrategia para abordar la educación alimentaria nutricional en el nivel inicial. +E. Revista de Extensión Universitaria, $8(9), 251-265$.

Camilloni, A. (2013). La inclusión de la educación experiencial en el currículo universitario. En G. Menéndez et al., Integración, docencia y extensión. Otra forma de enseñar y de aprender (pp. 11-21). Universidad Nacional del Litoral, Santa Fe.

Camilloni, A. (2017). Capítulo del libro. En G. Menéndez, Integración, docencia y extensión. Otra forma de enseñar y de aprender II. Universidad Nacional del Litoral, Santa Fe.

Cossani, Lopresti, Garnier, Reverdito, Tabárez, y Taffarel. (2011). La educación alimentaria nutricional como estrategia de articulación para favorecer la seguridad alimentaria en las familias de Entre Ríos. Integración, extensión, docencia e investigación para la inclusión y cohesión social. XI Congreso Iberoamericano de Extensión. Santa Fe, Argentina.

García, T., García, L., González, R., Carvalho, J. y Catarreira, S. (2016). Revisión metodológica de la triangulación como estrategia de investigación. CIAIQ, 3.

Gillespie, A. H. \& Johnson-Askew, W. (2009). Changing Family Food and Eating Practices: The Family Food Decision-making System. Annals of Behavioral Medicine, 38(suppl 1), s31-s36. doi:10.1007/s12160-009-9122-7 
Libera Bonilla, B. E. (2007). Impacto, impacto social y evaluación del impacto. ACIMED, 15(3), 1-9. http://scielo.sld.cu/scielo. php?script $=$ sci $\operatorname{arttext\& pid=S1024-94352007000300008}$

Maíz, E., Urdaneta, E. y Allirot, X. (2018). La importancia de involucrar a niños y niñas en la preparación de las comidas. Nutrición Hospitalaria, 35(Extra 4), 136-139. https://dx.doi.org/10.20960/nh.2139

Mayan, M. J. (2001). Una introducción a los métodos cualitativos: módulo de entrenamiento para estudiantes y profesionales. https://sites.ualberta. ca/ iiqm/pdfs/introduccion.pdf

Menéndez, G. et al. (Coords.). (2013). Integración, docencia y extensión. Otra forma de enseñar y de aprender. Universidad Nacional del Litoral, Santa Fe.

Minayo, M. C.; Assis, S. G. y Souza, E. R. (2008). Evaluación por triangulación de métodos. Abordaje de programas sociales. https://digitalrepository. unm.edu/lasm_cucs_es/146

Ministerio de Educación de Santa Fe. (2020). https://www.santafe.gov.ar/ index.php/educacion/guia/get tree by node?node_id $=121868$

Muñoz Razo, C. (1998). Cómo elaborar y asesorar una Investigación de tesis. Prentice Hall Hispanoamericana.

Ordoñana, J. R. (2003). Educación para la salud y comunicación sobre riesgos. En M. D., Flores (ed.), La promoción de la salud: una perspectiva pedagógica (pp. 41-57). Valencia: Nau Llibres.

Parrilla, Á; Muñoz-Cadavid, Ma A. y Sierra Martínez, S. (2013). Proyectos educativos con vocación comunitaria. Revista de Investigación en Educación, [S.1.], v. 11, n. 3, p. 15-31. ISSN 2172-3427. http://reined. webs.uvigo.es/index.php/reined/article/view/282

Rafaghelli, M. (2013). La dimensión pedagógica de la extensión. En G. Menéndez et al., Integración, docencia y extensión. Otra forma de enseñar y de aprender (pp. 22-37). Universidad Nacional del Litoral, Santa Fe.

Ravelli, S., Paniccia, A. y Figueroa, E. (2016). Desafíos de la educación alimentaria nutricional: construcción de espacios y vínculos en el nivel inicial. Revista $+E$, versión digital (6), 256-263. Santa Fe, Argentina: Ediciones UNL. 
Revista Universidad en Diálogo • Vol. 11, N. ${ }^{\circ}$ 1, Enero-Junio, 2021 • 157-178

ISSN 2215-2849 • EISSN: 2215-4752

URL: http://www.revistas.una.ac.cr/index.php/dialogo/index CORREO ELECTRÓNICO: universidadendialogo@una.cr DOI: http://doi.org/10.15359/udre.11-1.6

Universidad Nacional del Litoral. (2020). Proyectos de extensión. https:// www.fbcb.unl.edu.ar/vinculacion/proyectos-de-extension/

Vega, M. R., Manzanera, J. M. E. y Armenta, M. C. (2013). Una década enseñando e investigando en educación alimentaria para maestros/A decade teaching and researching in Food Education for Teachers. Revista Complutense de Educación, 24(2), 243-265. https://revistas. ucm.es/index.php/RCED/article/view/42078/40053 